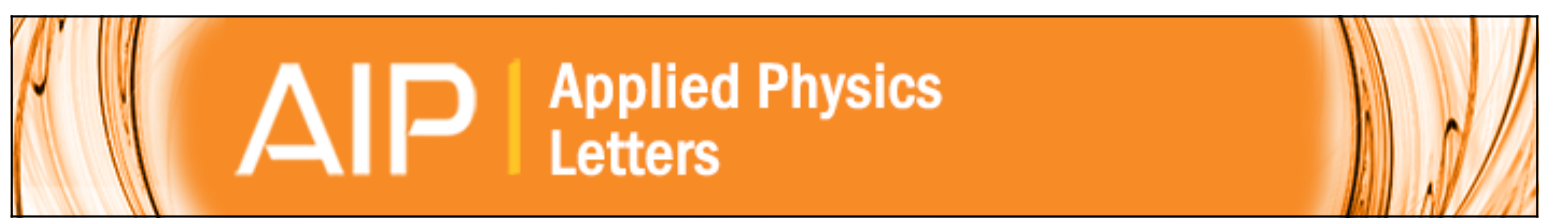

\title{
Unusual transport characteristics of nitrogen-doped single-walled carbon nanotubes
}

Yo-Sep Min, Eun Ju Bae, Un Jeong Kim, Eun Hong Lee, Noejung Park, Cheol Seong Hwang, and Wanjun Park

Citation: Applied Physics Letters 93, 043113 (2008); doi: 10.1063/1.2965805

View online: $h t t p: / / d x . d o i . o r g / 10.1063 / 1.2965805$

View Table of Contents: http://scitation.aip.org/content/aip/journal/apl/93/4?ver=pdfcov

Published by the AIP Publishing

\section{Articles you may be interested in}

Surface electronic structure of nitrogen-doped semiconducting single-walled carbon nanotube networks

J. Appl. Phys. 114, 153516 (2013); 10.1063/1.4826206

Electrical transport properties of boron-doped single-walled carbon nanotubes

J. Appl. Phys. 113, 054313 (2013); 10.1063/1.4790505

Controlling the doping of single-walled carbon nanotube networks by proton irradiation

Appl. Phys. Lett. 101, 103111 (2012); 10.1063/1.4751551

Effect of nitrogen-vacancy complex defects on the electronic transport of carbon nanotube Appl. Phys. Lett. 94, 102108 (2009); 10.1063/1.3082022

Electron doping of small-diameter carbon nanotubes with exohedral fullerenes Appl. Phys. Lett. 93, 223102 (2008); 10.1063/1.3041631

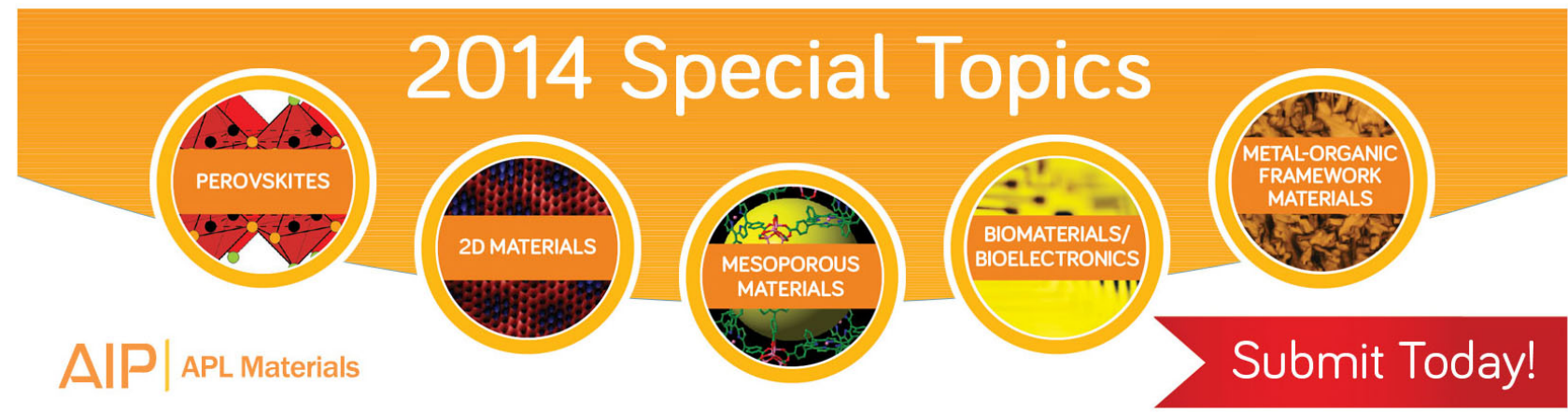




\title{
Unusual transport characteristics of nitrogen-doped single-walled carbon nanotubes
}

\author{
Yo-Sep Min, ${ }^{1}$ Eun Ju Bae, ${ }^{2}$ Un Jeong Kim, ${ }^{2}$ Eun Hong Lee, ${ }^{2}$ Noejung Park, ${ }^{3}$ \\ Cheol Seong Hwang, ${ }^{4}$ and Wanjun Park ${ }^{5, a)}$ \\ ${ }^{1}$ School of Chemical and Biological Engineering, Konkuk University, Seoul 143-701, Republic of Korea \\ ${ }^{2}$ Samsung Advanced Institute of Technology, Yong-In 449-712, Republic of Korea \\ ${ }^{3}$ Department of Applied Physics, Dankook University, Seoul 140-714, Republic of Korea \\ ${ }^{4}$ School of Materials Science and Engineering, Seoul National University, Seoul 151-742, Republic of Korea \\ ${ }^{5}$ Department of Electronics and Computer Engineering, Hanyang University, Republic of Korea
}

(Received 6 March 2008; accepted 2 July 2008; published online 29 July 2008)

\begin{abstract}
Electrical transport characteristics of nitrogen-doped single-walled carbon nanotubes (N-SWCNTs), in which the nitrogen dopant is believed to form a pyridinelike bonding configuration, are studied with the field effect transistor operations. Contrary to the expectation that the nitrogen atoms may induce a $n$-type doping, the electrical transports through our N-SWCNTs are either ambipolar in vacuum or $p$-type in air. Through the first-principles electronic structure calculations, we show that the nitrogen dopant indeed favors the pyridinelike configuration and the Fermi level of the pyridinelike N-SWCNT is almost at the intrinsic level. (c) 2008 American Institute of Physics. [DOI: $10.1063 / 1.2965805$ ]
\end{abstract}

Since the discovery of single-walled carbon nanotubes (SWCNTs) in 1993, its electrical transport properties have been intensely studied owing to the quasi-one-dimensional electron system as well as various potential applications. ${ }^{1}$ SWCNTs can be metallic or semiconducting, depending on a subtle change in the wrapping direction of the graphene sheet. ${ }^{2-5}$ While the metallic nanotube has been interesting because of its unprecedented large endurable current density, the semiconducting one has been more attractive as an ingredient in nanoscale electronic devices for future semiconductor technology. ${ }^{6-9}$

For the nanotube-based devices to be practically adopted in electronics, a full control of carrier type and carrier concentration is essentially required. In this regard, the charge transfer from/to the physically or chemically adsorbed species has been widely studied. Potassium or amine-containing molecules have been shown to induce the $n$-type characteristics of SWCNT-field effect transistors (FETs) ${ }^{10,11}$ Adsorption of environmental oxygen has been believed to be the origin of the common $p$-type behaviors of SWCNT-FETs. Oxygen molecules induce a hole doping in the nanotube channel as well as reduce the Schottky barrier at the metalnanotube contact. ${ }^{12-15}$

For a more stabilized control of carrier density in SWCNTs, substitution of foreign atoms may be preferred. While nitrogen atom has naturally been considered a good electron dopant, Czerw et al. ${ }^{16}$ reported that nitrogen dopants in the multiwalled bamboo structures constitute pyridinelike configurations, giving rise to strong electron donor states near the Fermi level. Zhao et al. ${ }^{17}$ also conjectured possible configurations of the nitrogen dopants in SWCNTs. They suggested that the nitrogen dopant, either in the form of a chemical adatom or the pyridinelike configuration, would lead to the $p$-type nanotubes, whereas the substitutional nitrogen dopant induces the $n$-type doping.

\footnotetext{
a) Author to whom correspondence should be addressed. Electronic mail: wanjun@hanyang.ac.kr.
}

Recently we reported that N-SWCNTs were directly grown on $\mathrm{SiO}_{2} / \mathrm{Si}$ substrates at $450{ }^{\circ} \mathrm{C}$ with methane and ammonia gases by water plasma chemical vapor deposition (CVD) ${ }^{18}$ It was characterized by x-ray photoelectron spectroscopy (XPS) that nitrogen atoms are doped with a pyridinelike configuration in our N-SWCNTs. ${ }^{18}$ In the present work, we report transport characteristics of the N-SWCNTFET employing the pyridinelike N-SWCNTs as a transport channel. Through the first-principles total energy calculations, we show that the pyridinelike nitrogen configuration is indeed energetically favored. Our N-SWCNTs show an ambipolar and $p$-type transport behaviors in vacuum and in air, respectively. Such transport characteristics are discussed in terms of the electronic structures, depending on the bonding configurations of the nitrogen dopants.

For the fabrication of the back-gate transistors, the network of N-SWCNTs with a nitrogen concentration of $\sim 0.6 \%$ was site selectively grown with an ammonia flow of 1 SCCM (SCCM denotes cubic centimeter per minute at STP) in defined regions for channel on $\mathrm{SiO}_{2}(400 \mathrm{~nm}) / \mathrm{Si}$ substrates by the direct photolithographic method previously reported. ${ }^{19}$ Source and drain electrodes of $\mathrm{Ti}(10 \mathrm{~nm}) / \mathrm{Au}$ $(100 \mathrm{~nm})$ were formed by e-beam evaporation on the nanotube-grown substrate and subsequent lift-off process. The fabricated network transistors have a channel length of $2 \mu \mathrm{m}$ and a width of $40 \mu \mathrm{m}$. The electrical measurements were performed at room temperature in air and in vacuum $\left(\sim 10^{-6}\right.$ Torr $)$ with a semiconductor parameter analyzer (HP4156C). The positive and negative regions of the gate voltage in Fig. 1 were swept from 0 to 30 and $-30 \mathrm{~V}$, respectively, and combined.

In Fig. 1(a), the transistor of N-SWCNT network shows $p$-type transport behavior in air, as characterized by the onand off-states in negative and positive biases, respectively, with the $I_{\text {on }} / I_{\text {off }}$ ratio of $10^{4}-10^{5}$. However, the $p$-type unipolar characteristics change to ambipolar transport only after evacuation for several hours, as shown in Fig. 1(b). It should be noted that the large increases in $I_{\mathrm{ds}}$ at high $V_{\mathrm{g}}$ are not due 

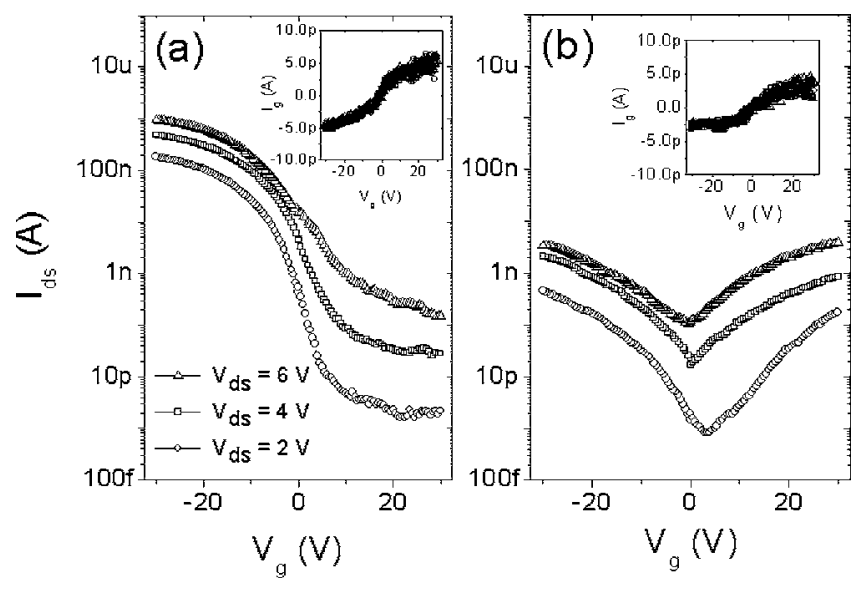

FIG. 1. Drain-source current $\left(I_{\mathrm{ds}}\right)$ versus gate voltage $\left(V_{\mathrm{g}}\right)$ characteristics of the network transistor of N-SWCNTs in (a) ambient air and in (b) vacuum $\left(\sim 10^{-6}\right.$ Torr). The inset figures show the leakage currents $\left(I_{\mathrm{g}}\right)$ vs $V_{\mathrm{g}}$ curves in air and in vacuum, respectively.

to the leakage currents $\left(I_{\mathrm{g}}\right)$ through the gate oxide as compared in the inset figures. Such a conversion from $p$-type to ambipolar behavior is a typical behavior of the intrinsic SWCNT transistors, being ascribed to the removal of environmental oxygens. ${ }^{15}$ The ambipolar behavior of our $\mathrm{N}$-SWCNTs under vacuum condition suggests that the nitrogen dopants in our sample do not donate electron carrier to the conduction band of the SWCNT. Previously, based on XPS data, we discussed that the nitrogen dopants constitute the pyridinelike configuration in our N-SWCNT. ${ }^{18}$ These are consistent with our theoretical results which will be presented in the later paragraph.

Now we perform the first-principles density functional calculations to investigate the formation energy as well as the electronic structure of the N-SWCNTs. We use the Vienna $a b$ initio simulation package, ${ }^{20,21}$ where the PerdewBurke-Enzerh-type generalized gradient approximation is

(a)

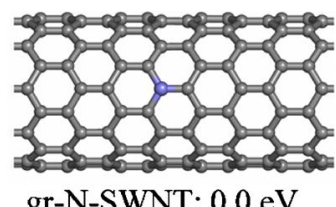

(b)

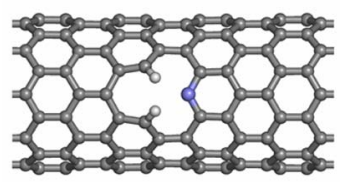

pr-N-SWNT1: $-0.63 \mathrm{eV}$

(c)

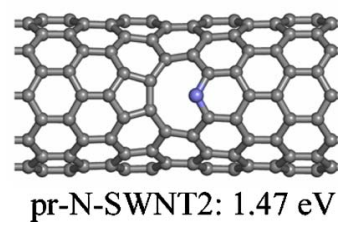

FIG. 2. (Color online) Geometries for the N-SWCNTs in (a) the graphitelike configuration and the pyridinelike configurations (b) with and (c) without passivating hydrogen atoms. Dark gray, blue, and white spheres stand for carbon, nitrogen, and hydrogen atoms, respectively. Relative energetics according to the formula described in the text is presented in eV unit. Shown geometries are optimized by the energy minimization calculations. employed for the exchange correlation potential, ${ }^{22}$ and the projector augmented wave method is used for ionic potential. ${ }^{23}$ The plane wave basis set, with the kinetic energy cutoff of $400 \mathrm{eV}$, is employed to describe the Kohn-Sham wave functions. We choose the $(10,0)$ nanotube as a representative case for the semiconducting carbon nanotube.

Figure 2 shows atomic configurations of the N-SWCNTs. The graphitelike configuration, in which the nitrogen dopant substitutes one carbon atom, is denoted by gr-N-SWCNT, as shown in Fig. 2(a). Here we investigate two types of the pyridinelike configurations. When two hydrogen atoms are attached to passivate the broken carbon bond, as shown in Fig. 2(b), the configuration is designated as pr-N-SWCNT1. Without such hydrogen atoms the total energy minimization calculation resulted in the formation of carbon pentagonal ring, as shown in Fig. 2(c), which is denoted by pr-N-SWCNT2. We note that there may be significant presences of methane, ammonia, and hydrogen molecules near the growing edge of the SWCNT during the CVD process. Then the energy change in the following transformation is likely to be a good indicator for the relative stability between N-SWCNTs:

$$
\begin{aligned}
& \text { gr-N-SWCNT }+3 \mathrm{H}_{2} \rightarrow \text { pr-N-SWCNT1 }+\mathrm{CH}_{4}, \\
& \text { gr-N-SWCNT }+2 \mathrm{H}_{2} \rightarrow \text { pr-N-SWCNT2 }+\mathrm{CH}_{4} .
\end{aligned}
$$

The energy changes are presented in Fig. 2. The geometry shown in Fig. 2 is the computational unit cell and the formation energy measured in this way tells that one pyridinelike configuration (pr-N-SWCNT1), described in Fig. 2(b), is indeed energetically favored than the graphitelike configuration.

In Fig. 3, we compare the electronic structure of the pure $(10,0)$ SWCNT [Fig. 3(a)], the N-SWCNT in the graphitelike configurations [Fig. 3(b)], and those in pyridinelike configurations [Fig. 3(c) and 3(d)]. Figure 3(a) shows the partial density of states (DOS) for 20 carbon atoms along the zigzag chain of the pure $(10,0)$ SWCNT. The two downward arrows indicate the valence band and conduction band edges of the SWCNT, respectively. In the case of the graphitelike nitrogen doping, as shown in Fig. 3(b), the Fermi level locates at the conduction band edge of the N-SWCNT. We note that the nitrogen-derived states are much overlapped with the carbon states, and the $\pi$ electron states in the conduction bands are substantially occupied. This obviously represents the electron-donating features of the graphiticlike nitrogen dopant, being in consistent with many previous reports. ${ }^{17,24}$

However, in the case of the pyridinelike nitrogen doping, as shown in Fig. 3(c), we observe that the Fermi level is now at the midgap of the nanotube. This type of nitrogen dopant does not contribute to electron carriers, and the transport measurement should result in an ambipolar behavior, as consistent with our measurements. In this $(10,0)$ nanotube, the pyridine ring does not necessarily align with the nanotube axis. Two different configurations could be constructed by rotating the pyridine ring by $60^{\circ}$ in its plane. We found that overall features of the electronic structure of such pyridinelike N-SWCNTs were almost the same, as that shown in Fig. $3(\mathrm{c})$. This implies that the orientation of the pyridine ring does not matter, and further the effect of the pyridinelike $\mathrm{N}$ doping in nanotubes of different chiralities should be nearly the same. Figure 3(d) shows the electronic structure of the 


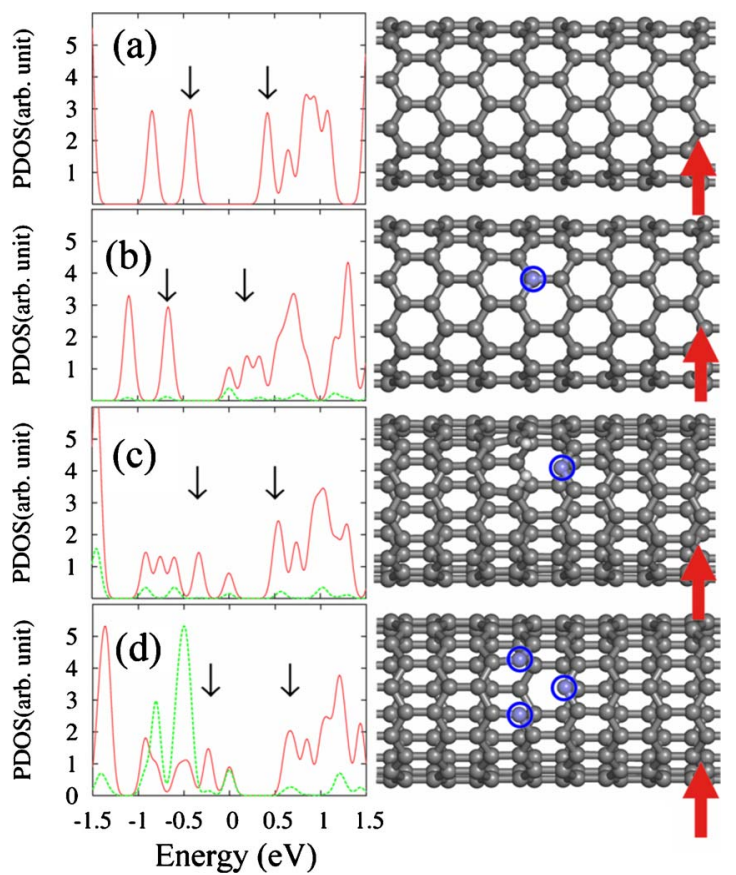

FIG. 3. (Color online) Electronic structure of the $(10,0)$ nanotubes (a) without any defect, (b) with one graphitelike $\mathrm{N}$ dopant, (c) with one pyridinelike $\mathrm{N}$ dopant and two passivating hydrogen atoms, and (d) with three neighbored pyridinelike $\mathrm{N}$ dopants. Solid red lines are DOSs for the carbon atom in the zigzag chain (20 C atoms) and the dotted green lines are those of nitrogen atoms. Two downward arrows in (a)-(d) indicate the position of the valence band and conduction band edges of the intrinsic carbon nanotube. Corresponding atomic geometries, which are the unit cell in the ab initio calculations, are shown right to the DOSs. In the atomic geometries nitrogen atoms are emphasized with blue circles. Upward thick arrow in the atomic geometry indicates the selected carbon zigzag chains for the partial DOS plot.

N-SWCNT with three neighboring pyridinelike nitrogen dopants. In this case the Fermi level is also in the gap of the nanotube, but closer to the valence band edge of the SWCNT.

In summary, electrical transport characteristics of $\mathrm{N}-\mathrm{SWCNTs}$ with a pyridinelike bonding configuration was studied with the FET operations. Contrary to the expectation for the $n$-type doping nature of nitrogen atom, the electrical transports through our N-SWCNTs are $p$-type in air and ambipolar in vacuum. The electronic structure calculations showed that the Fermi level of the N-SWCNT with such a pyridinelike nitrogen dopant is almost at the intrinsic level, which is quite in contrast to the obvious electron-donating feature of the nitrogen dopant in graphitelike configuration, and explains well the intrinsiclike electrical behavior of our N-SWCNTs.

This work was supported by the Tera-level NanoDevices (TND) Program of the Ministry of Science and Technology, Korea.

${ }^{1}$ M. S. Dresselhaus, G. Dresselhaus, and Ph. Avouris, Carbon Nanotubes: Synthesis, Structure, Properties and Applications (Springer, Berlin, 2001).

${ }^{2}$ J. W. Mintmire, B. I. Dunlap, and C. T. White, Phys. Rev. Lett. 68, 631 (1992).

${ }^{3}$ N. Hamada, S. Sawada, and A. Oshiyama, Phys. Rev. Lett. 68, 1579 (1992).

${ }^{4}$ R. Saito, M. Fujita, G. Dresselhaus, and M. S. Dresselhaus, Appl. Phys. Lett. 60, 2204 (1992).

${ }^{5}$ T. W. Odom, J. L. Huang, P. Kim, and C. M. Lieber, Nature (London) 391, 62 (1998).

${ }^{6} \mathrm{Ph}$. Avouris, Acc. Chem. Res. 35, 1026 (2002).

${ }^{7}$ S. J. Tans, A. R. M. Verschueren, and C. Dekker, Nature (London) 393, 49 (1998).

${ }^{8}$ A. Javey, J. Guo, Q. Wang, M. Lundstrom, and H. Dai, Nature (London) 424, 654 (2003).

${ }^{9}$ Z. Chen, J. Appenzeller, Y. Lin, J. Sippel-Oakley, A. G. Rinzler, J. Tang, S. J. Wind, P. M. Solomon, and Ph. Avouris, Science 311, 1735 (2006).

${ }^{10}$ A. Javey, R. Tu, D. B. Farmer, J. Guo, R. G. Gordon, and H. Dai, Nano Lett. 5, 345 (2005).

${ }^{11}$ C. Klinke, J. Chen, A. Afzali, and Ph. Avouris, Nano Lett. 5, 555 (2005).

${ }^{12}$ V. Derycke, R. Martel, J. Appenzeller, and Ph. Avouris, Appl. Phys. Lett. 80, 2773 (2002).

${ }^{13}$ S. H. Jhi, S. G. Louie, and M. L. Cohen, Phys. Rev. Lett. 85, 1710 (2000).

${ }^{14}$ M. H. Yang, K. B. K. Teo, W. I. Milne, and D. G. Hasko, Appl. Phys. Lett. 87, 253116 (2005).

${ }^{15}$ D. Kang, N. Park, J. Hyun, E. J. Bae, J. Ko, J. Kim, and W. Park, Appl. Phys. Lett. 86, 093105 (2005).

${ }^{16}$ R. Czerw, M. Terrones, J. C. Charlier, X. Blasé, B. Foley, R. Kamalakaran, N. Grobert, H. Terrones, D. Tekleab, P. M. Ajayan, W. Blau, M. Ruhle, and D. L. Carroll, Nano Lett. 1, 457 (2001).

${ }^{17}$ M. Zhao, Y. Xia, J. P. Lewis, and R. Zhang, J. Appl. Phys. 94, 2398 (2003).

${ }^{18}$ Y. S. Min, E. J. Bae, I. P. Asanov, U. J. Kim, and W. Park, Nanotechnology 18, 285601 (2007).

${ }^{19}$ Y. S. Min, E. J. Bae, J. B. Park, and W. Park, Nanotechnology 17, 116 (2006).

${ }^{20} \mathrm{G}$. Kresse and J. Hafner, Phys. Rev. B 49, 14251 (1994).

${ }^{21}$ G. Kresse and J. Furthmüller, Comput. Mater. Sci. 6, 15 (1996).

${ }^{22}$ J. P. Perdew, K. Burke, and M. Ernzerhof, Phys. Rev. Lett. 77, 3865 (1996).

${ }^{23}$ G. Kresse and D. Joubert, Phys. Rev. B 59, 1758 (1999).

${ }^{24}$ H. S. Kang and S. Jeong, Phys. Rev. B 70, 233411 (2004). 